Jie Cheng and Jue Chen*

\title{
The crystal structure of 1,2-bis(4-pyridyl)ethane - 4,4-dihydroxydiphenylmethane (1/1), $\mathrm{C}_{25} \mathrm{H}_{21} \mathrm{~N}_{2} \mathrm{O}_{2}$
}

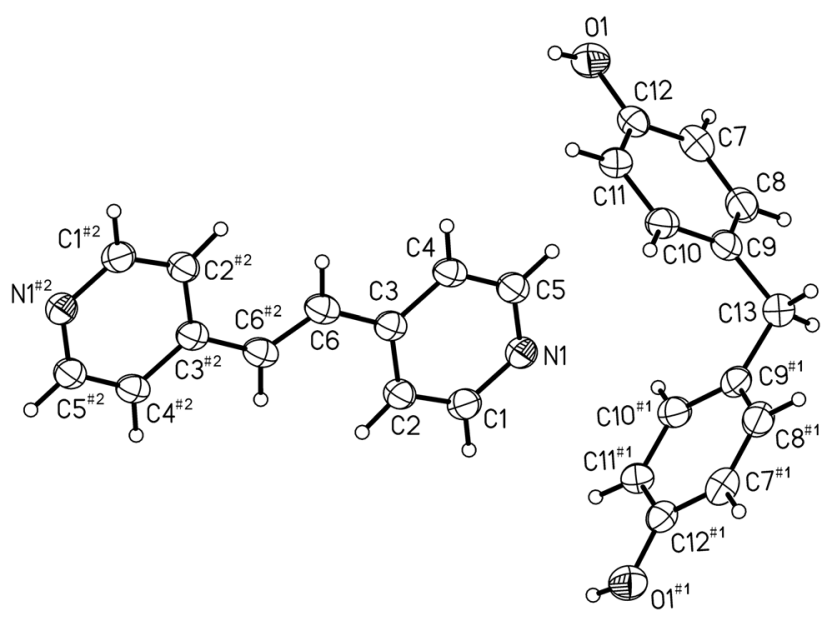

https://doi.org/10.1515/ncrs-2021-0148

Received April 18, 2021; accepted April 29, 2021;

published online May 13, 2021

Table 1: Data collection and handling.

\begin{tabular}{ll}
\hline Crystal: & Colorless block \\
Size: & $0.41 \times 0.23 \times 0.15 \mathrm{~mm}$ \\
Wavelength: & Mo $K \alpha$ radiation $(0.71073 \AA$ A \\
$\mu:$ & $0.08 \mathrm{~mm}^{-1}$ \\
Diffractometer, scan mode: & Rigaku R-AXIS RAPID, $\omega$ \\
$\theta_{\text {max }}$, completeness: & $25.0^{\circ},>99 \%$ \\
$N(h k l)_{\text {measured }} N(h k l)_{\text {unique }}, R_{\text {int }}:$ & $7912,1833,0.046$ \\
$C$ riterion for $I_{\text {obs }}, N(h k l)_{\text {gt }}:$ & $I_{\text {obs }}>2 \sigma\left(I_{\text {obs }}\right), 1121$ \\
$N(\text { param })_{\text {refined }}:$ & 132 \\
Programs: & RAPID-AUTO [1], SHELX [2, 3]
\end{tabular}

methanol-water $(\mathrm{v}: \mathrm{v}=1: 1)$. The solution was allowed to stand at room temperature for three days, colorless block crystals were obtained.

\section{Experimental details}

The structure was solved by direct methods with the SHELXS program. All $\mathrm{H}$-atoms at $\mathrm{C}$ atoms were positioned with idealized geometry and refined isotropically $\left(U_{i s o}(\mathrm{H})=1.2 U_{e q}(\mathrm{C})\right.$, using a riding model with $\mathrm{C}-\mathrm{H}=0.93 \AA$ or $0.96 \AA$ A $\mathrm{H}$ atoms attached to $\mathrm{O}$ atoms were found in a difference Fourier synthesis and were refined using a riding model, with the $\mathrm{O}-\mathrm{H}$ distances fixed as initially found and with $U_{\text {iso }}(\mathrm{H})$ values set at $1.5 U_{e q}(\mathrm{O})$.

\section{Comment}

Organic cocrystals, composed of two or more molecular and/or ionic compounds in a stoichiometric ratio, are crystalline or single-phase materials [4, 5]. Over the past decades, they have attracted wide attention and emerged as a promising research interest to construct functional materials due to their unique electrical, magnetic, and optical properties [6-8]. Crystallization is a typical selfassembly phenomenon [9]. The synergistic effect of those non-covalent bonding interactions between homomeric and heteromeric molecules governs recognition and assembly process [10-14]. The synthon that is formed

\footnotetext{
*Corresponding author: Jue Chen, School of Biological and Chemical Engineering, NingboTech University, Ningbo, 315100, People's Republic of China, E-mail: chj@nit.zju.edu.cn. https://orcid.org/ 0000-0002-7516-9842

Jie Cheng, School of Biological and Chemical Engineering, NingboTech University, Ningbo, 315100, People's Republic of China
} 
Table 2: Fractional atomic coordinates and isotropic or equivalent isotropic displacement parameters $\left(\AA^{2}\right)$.

\begin{tabular}{lrrrr}
\hline Atom & $\boldsymbol{x}$ & $\boldsymbol{y}$ & $\boldsymbol{z}$ & $\boldsymbol{U}_{\text {iso }} / \boldsymbol{U}_{\text {eq }}$ \\
\hline N1 & $0.68915(17)$ & $0.5103(2)$ & $0.56133(9)$ & $0.0827(6)$ \\
O1 & $0.80659(16)$ & $0.7771(2)$ & $0.61794(8)$ & $0.1045(6)$ \\
H1B & 0.769426 & 0.699137 & 0.601436 & $0.157^{*}$ \\
C1 & $0.6486(2)$ & $0.3846(3)$ & $0.59222(10)$ & $0.0889(7)$ \\
H1A & 0.659699 & 0.395780 & 0.630723 & $0.107^{*}$ \\
C2 & $0.5922(2)$ & $0.2410(3)$ & $0.57102(10)$ & $0.0812(7)$ \\
H2A & 0.565853 & 0.158329 & 0.594726 & $0.097^{*}$ \\
C3 & $0.57448(18)$ & $0.2197(3)$ & $0.51376(9)$ & $0.0687(6)$ \\
C4 & $0.61564(18)$ & $0.3507(3)$ & $0.48175(9)$ & $0.0722(6)$ \\
H4A & 0.606149 & 0.342875 & 0.443136 & $0.087^{*}$ \\
C5 & $0.6698(2)$ & $0.4905(3)$ & $0.50641(11)$ & $0.0773(7)$ \\
H5A & 0.694653 & 0.576915 & 0.483673 & $0.093^{*}$ \\
C6 & $0.51960(18)$ & $0.0680(3)$ & $0.48763(9)$ & $0.0764(6)$ \\
H6A & 0.511212 & 0.067009 & 0.448926 & $0.092^{*}$ \\
C7 & $0.7773(3)$ & $0.9987(3)$ & $0.68096(11)$ & $0.0930(8)$ \\
H7A & 0.858162 & 1.030358 & 0.679906 & $0.112^{*}$ \\
C8 & $0.7024(3)$ & $1.0884(3)$ & $0.71386(10)$ & $0.0906(8)$ \\
H8A & 0.734620 & 1.179410 & 0.734986 & $0.109^{*}$ \\
C9 & $0.5825(2)$ & $1.0465(3)$ & $0.71606(9)$ & $0.0811(7)$ \\
C10 & $0.5384(2)$ & $0.9080(3)$ & $0.68516(10)$ & $0.0828(7)$ \\
H10A & 0.457515 & 0.876246 & 0.686172 & $0.099^{*}$ \\
C11 & $0.6120(2)$ & $0.8153(3)$ & $0.65273(9)$ & $0.0787(6)$ \\
H11A & 0.580689 & 0.721214 & 0.632911 & $0.094^{*}$ \\
C12 & $0.7308(2)$ & $0.8619(3)$ & $0.64976(10)$ & $0.0780(6)$ \\
C13 & 0.500000 & $1.1501(4)$ & 0.750000 & $0.0995(11)$ \\
H13A & 0.450320 & 1.222249 & 0.725140 & $0.119^{*}$ \\
\hline & & & &
\end{tabular}

between bisphenol and other moieties is one of the most exploted synthon for designing cocrystal [15-19].

The asymmetric unit of the title structure contains one half of a 4,4'-dihydroxydiphenylmethane molecule and one half of a 1,2-bis(4-pyridyl)ethane molecule (see the figure). Bond lengths and angles are within normal ranges. In the crystal structure of the title complex, $\mathrm{O}-\mathrm{H} \cdots \mathrm{N}$ hydrogen interactions $\left(\mathrm{O} 1 \cdots \mathrm{N} 1=2.759(6) \AA\right.$; $\left.\mathrm{O} 1-\mathrm{H} 1 \mathrm{~B} \cdots \mathrm{N} 1=177.1^{\circ}\right)$ between the 4,4'-dihydroxydiphenylmethane and 1,2-bis (4-pyridyl)ethane molecules lead to the formation of a chain along the $c$ axis. Two pyridine groups of neighboring molecules, related by inversion symmetry are involved in a $\pi-\pi$ interaction with a centroid-centroid distance of $3.78 \AA$ forming a two-dimensional layer structure parallel to (100).

Author contributions: All the authors have accepted responsibility for the entire content of this submitted manuscript and approved submission.

Research funding: None declared.

Conflict of interest statement: The authors declare no conflicts of interest regarding this article.

\section{References}

1. Rigaku. RAPID-AUTO; Rigaku Corporation: Tokyo, Japan, 1998.

2. Sheldrick G. M. SHELXTL - integrated space-group and crystalstructure determination. Acta Crystallogr. 2015, A71, 3-8.

3. Sheldrick G. M. Crystal structure refinement with SHELXL. Acta Crystallogr. 2015, C71, 3-8.

4. Park S. K., Varghese S., Kim J. H., Yoon S. J., Kwon O. K., An B. K., Gierschner J. Tailor-made highly luminescent and ambipolar transporting organic mixed stacked charge-transfer crystals: an isometric donor-acceptor approach. J. Am. Chem. Soc. 2013, 135, 4757-4764.

5. Wu H. D., Wang F. X., Xiao Y., Pan G. B. Preparation of nano/ microstructures of CUOEP-TCNQ cocrystals with controlled stacking and their photoresponse properties. J. Mater. Chem. C 2014, 2, 2328-2332.

6. Park S. K., Kim J. H., Park S. Y. Organic 2D optoelectronic crystals: charge transport, emerging functions, and their design perspective. Adv. Mater. 2018, 30, 1704759.

7. Sun L. J., Zhu W. G., Yang F. X., Li B. L., Ren X. C., Zhang X. T., Hu W. P. Molecular cocrystals: design, charge-transfer and optoelectronic functionality. Phys. Chem. Chem. Phys. 2018, 20, 6009-6023.

8. Sun L. J., Wang Y., Yang F. X., Zhang X. T., Hu W. P. Cocrystal engineering: a collaborative strategy toward functional materials. Adv. Mater. 2019, 31, 1902328.

9. Zhu W. G., Zheng R. H., Fu X. L., Fu H. B., Shi Q., Zhen Y. G., Dong H. L., Hu W. P. Revealing the charge-transfer interactions in selfassembled organic cocrystals: two-dimensional photonic applications. Angew. Chem. 2015, 127, 6889-6893.

10. Zhang J., Xu W., Sheng P., Zhao G. Y., Zhu D. B. Organic donoracceptor complexes as novel organic semiconductors. Acc. Chem. Res. 2017, 50, 1654-1662.

11. Harada J., Ohtani M., Takahashi Y., Inabe T. Molecular motion, dielectric response, and phase transition of charge-transfer crystals: acquired dynamic and dielectric properties of polar molecules in crystals. J. Am. Chem. Soc. 2015, 137, 4477-4486.

12. Ong T. T., Kavuru P., Nguyen T., Cantwell R., Wojtas L., Zaworotko M. J. 2:1 cocrystals of homochiral and achiral amino acid zwitterions with $\mathrm{Li}^{+}$salts: water-stable zeolitic and diamondoid metal-organic materials. J. Am. Chem. Soc. 2011, 133, 9224-9227.

13. Huang Y. J., Gong Q. Y., Ge J., Tang P. P., Yu F., Xiao L., Wang Z. R., Sun H. D., Yu J., Li D. S., Xiong Q. H., Zhang Q. C. Green grindingcoassembly engineering toward intrinsically luminescent tetracene in cocrystals. ACS Nano 2020, 14, 15962-15972.

14. Mazzeo P. P., Carraro C., Monica A., Capucci D., Pelagatti P., Bianchi F., Agazzi S., Careri M., Raio A., Carta M., Menicucci F., Belli M., Michelozzi M., Bacchi A. Designing a palette of cocrystals based on essential oil constituents for agricultural applications. ACS Sustain. Chem. Eng. 2019, 7, 17929-17940.

15. Zhang Z. H., Tan X., Chen S. C. 4,4'-Methylenediphenol4,4'-bipyridine (2/3): decarboxylation of 5,5'-methylenedisalicylic acid under hydrothermal conditions. Acta Crystallogr. 2009, C65, 0457-0459.

16. Iwase N., Kinuta T., Tajima N., Stao T., Kuroda R., Matsubara Y., Imai Y. Molecular recognition of bisphenol $A$ and its derivatives using p-benzoquinone. CrystEngComm 2010, 12, 3195-3200.

17. Vangla V. R., Mondal R., Broder C. K., Howard J. A. K., Desiraju G. R. Dianiline-diphenol molecular complexes 
based on supraminol recognition. Cryst. Growth Des. 2005, 5, 99-104.

18. Lim C. F., Tanski J. M. Structural analysis of bisphenol-A and its methylene, sulfur, and oxygen bridged bisphenol analogs.

J. Chem. Crystallogr. 2007, 37, 587-595.
19. Yang Y. X., Li Q. Hydrogen-bonded molecular ladders and interlaced networks in complexes built of $\mathrm{V}$-shaped molecules 4,4'-isopropylidenediphenol or 4,4'-oxydibenzoic acid with 4,4'-bipyridine. J. Chem. Crystallogr. 2011, 41, 929-935. 